\title{
Impact of the Sensitivity to Empiric Antibiotics on Clinical Outcomes after Biliary Drainage for Acute Cholangitis
}

\author{
Satoshi Kawamura ${ }^{1}$, Yuki Karasawa ${ }^{1}$, Nobuo Toda ${ }^{1}$, Yousuke Nakai ${ }^{2}$, Chikako Shibata $^{1}$, Ken Kurokawa $^{1}$, Junya $_{\text {Arai }}{ }^{1}$, \\ Kazuyoshi Funato ${ }^{1}$, Shigeyuki Kurosaki ${ }^{1}$, Shuya Maeshima ${ }^{1}$, Mayuko Kondo ${ }^{1}$, Kentaro Kojima ${ }^{1}$, Takamasa Ohki ${ }^{1}$, \\ Michiharu Seki ${ }^{1}$, Kazuhiko Koike ${ }^{2}$, and Kazumi Tagawa ${ }^{1}$ \\ ${ }^{1}$ Department of Gastroenterology, Mitsui Memorial Hospital, and ${ }^{2}$ Department of Gastroenterology, University of Tokyo Hospital, Tokyo, Japan
}

Background/Aims: Empiric antibiotics are given in combination with biliary drainage for acute cholangitis but sometimes turn out to be insensitive to microorganisms in blood and bile. Clinical outcomes were compared according to sensitivity to microorganisms detected in blood and bile culture to evaluate the impact of sensitivity to empiric antibiotics in cholangitis. Methods: Consecutive patients who underwent biliary drainage for acute cholangitis were retrospectively studied. Clinical outcomes such as 30-day mortality, length of hospital stay and high care unit stay, organ dysfunction and duration of fever were compared in three groups: group A (sensitive to both blood and bile culture), group B (sensitive to blood culture alone) and group C (insensitive to both blood and bile culture). Results: Eighty episodes of cholangitis were classified according to sensitivity results: 42, 32 and six in groups A, B and C. Escherichia coli and Klebsiella were two major pathogens. There were no significant differences in 30-day mortality rate $(7 \%, 0 \%$, and $0 \%, p=0.244)$, length of hospital stay $(28.5,21.0$, and 20.5 days, $p=0.369)$, organ dysfunction rate $(14 \%, 25 \%$, and $17 \%, p=0.500)$, duration of fever (4.3, 3.2, and 3.5 days, $p=0.921)$ and length of high care unit stay $(1.4,1.2$, and 1.7 days, $p=0.070)$ in groups $A$, $B$ and $C$. Empiric antibiotics were changed in 11 episodes but clinical outcomes appeared to be non-inferior even in 31 episodes of cholangitis who were on inadequate antibiotics throughout the course. Conclusions: Sensitivity of empiric antibiotics was not associated with clinical outcomes in acute cholangitis. (Gut Liver 2020;14:842-849)

Key Words: Endoscopic retrograde cholangiopancreatography; Cholangitis; Antimicrobial agents

\section{INTRODUCTION}

Acute cholangitis is caused by bacterial infection in the obstructed biliary system due to choledocholithiasis, benign biliary stricture or malignancy and the mortality rate in severe acute cholangitis is reportedly as high as $20 \% .{ }^{1}$ Treatment for acute cholangitis is a combination of biliary drainage and antibiotics $^{2-5}$ and empiric broad spectrum antibiotics are recommended for cholangitis. However, empiric antibiotics sometimes fail to cover microorganisms subsequently detected in blood or bile culture due to the increase in resistant pathogens in cholangitis. ${ }^{6}$ While the use of insensitive antimicrobial treatment for bloodstream infections is often related to worse clinical outcomes, ${ }^{7-11}$ conflicting data are reported about the associations between sensitivity of empiric antibiotics and mortality in cholangitis, ${ }^{6,12,13}$ depending on the successful biliary drainage. ${ }^{14}$ Furthermore, microorganisms identified in blood and bile culture sometimes differ in acute cholangitis, though bile culture is more often polymicrobial than blood culture. However, it is still unclear whether sensitivity to microorganisms in blood and/or bile affects clinical outcomes in patients with successful biliary drainage for acute cholangitis. ${ }^{15-17}$

Therefore, we conducted this retrospective analysis to compare clinical outcomes according to sensitivity to microorganisms detected in blood and bile culture.

\section{MATERIALS AND METHODS}

\section{Study design and population}

This was a single center, retrospective study of patients undergoing biliary drainage for acute cholangitis in a Japanese secondary care hospital. Data on consecutive patients who

Correspondence to: Nobuo Toda

Department of Gastroenterology, Mitsui Memorial Hospital, Kanda-Izumi-cho 1, Chiyoda-ku, Tokyo 101-8643, Japan

Tel: +81-3-3862-9111, Fax: +81-3-3864-7901, E-mail: ntoda@mitsuihosp.or.jp

Received on May 18, 2019. Revised on October 20, 2019. Accepted on November 3, 2019. Published online February 14, 2020.

pISSN 1976-2283 eISSN 2005-1212 https://doi.org/10.5009/gnl19248

@ This is an Open Access article distributed under the terms of the Creative Commons Attribution Non-Commercial License (http://creativecommons.org/licenses/by-nc/4.0) which permits unrestricted non-commercial use, distribution, and reproduction in any medium, provided the original work is properly cited. 
underwent biliary drainage within 24 hours of the diagnosis of acute cholangitis from January 2010 and December 2015 were extracted from the electrical medical records. Patients with positive blood and/or bile culture were included in our analysis. Patients whose sensitivity analysis of blood and bile culture were not available were excluded from the analysis. Collected data were as follows: age, sex, comorbidity, laboratory data including microorganism evaluation, severity and causes of cholangitis, biliary drainage methods, the timing of cholangitis (nosocomial or not), prior history of biliary drainage, hospitalization (within 30 days) and antibiotics use (within 30 days). This study was approved by the Ethical Committee at Mitsui Memorial Hospital (IRB number: MEC2018-C12).

\section{Microbiological assessment and antibiotics use}

Bile aspirated during endoscopic retrograde cholangiopancreatography (ERCP) or percutaneous transhepatic biliary drainage was sent for culture. Blood culture was obtained at separate two sites before administration of antibiotics. One set of positive blood culture for skin inhabitants (i.e., Staphylococcus epidermis, Corynebacterium or Bacillus species) was considered contamination.

In general, sulbactam/cefoperazone (SBT/CPZ) was used as the initial empiric antimicrobial therapy to cover gram negative rods and aerobes. Ampicillin was added in patients who were considered as at high risk of Enterococcus spp. infection such as a history of sphincterotomy or an indwelling biliary stent. In cases with a history of ampicillin-resistant Enterococcus spp. infection, vancomycin was added to SBT/CPZ. In cases with a deteriorated general condition, meropenem or doripenem was selected to cover extended-spectrum $\beta$-lactamase producing Escherichia coli or Klebsiella spp. Meropenem was also selected in some patients as the initial antibiotics based on the sensitivity analysis of microorganisms detected in the bile aspirate at the previous interventions. Ciprofloxacin or levofloxacin was administrated in patients who were allergic to penicillin or cephalosporin, respectively.

Initial empiric antibiotics were considered as sensitive if they were sensitive to all the microorganisms isolated from blood and/or bile culture. Antibiotics was changed at the discretion of the physicians in cases whose blood or bile culture showed microorganisms insensitive to the empiric antibiotics or in cases without clinical response defined as resolution of fever and jaundice despite successful biliary drainage.

\section{Study outcomes}

The aim of this study was to evaluate the associations of sensitivity of blood and bile culture with clinical outcomes after successful biliary drainage in patients with acute cholangitis. Patients were categorized into three groups according to sensitivity to the initial empiric antibiotics. The initial empiric antibiotics were sensitive to microorganisms in blood and bile culture (group A), sensitive to microorganisms in blood culture alone (group B) and not sensitive to microorganisms in blood and bile culture (group C). The primary outcome is 30-day mortality and secondary outcomes are the length of hospital stay, the incidence of organ dysfunction, the duration of fever after antibiotics use and the length of high care unit stay.

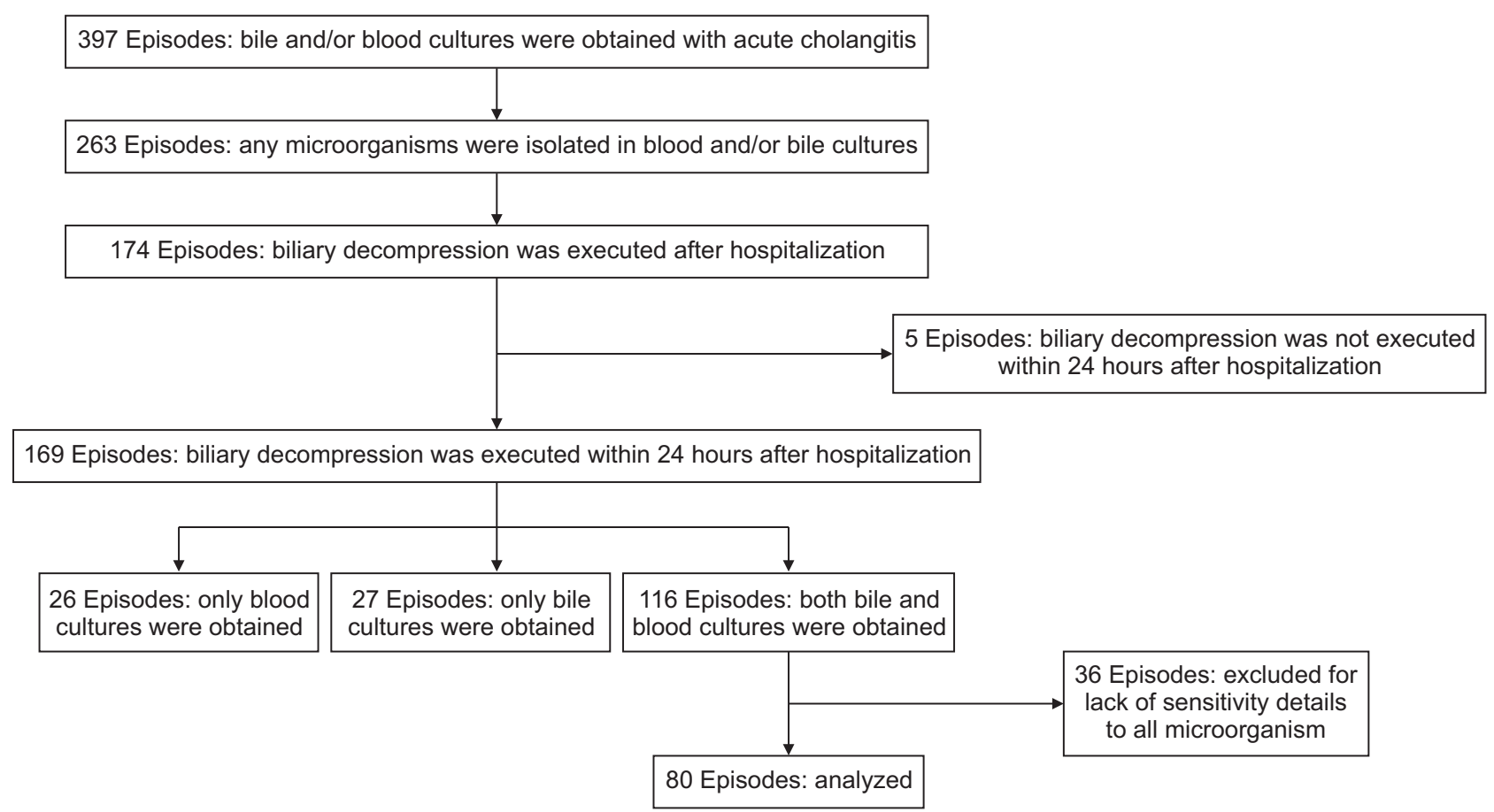

Fig. 1. Patient flowchart. 


\section{Definitions}

The diagnosis and severity assessment of acute cholangitis including organ dysfunction was based on TG13. ${ }^{18}$ The duration of fever was the period between initiation of antibiotics and the day when a body temperature of $<37^{\circ} \mathrm{C}$ was maintained for 24 hours. ${ }^{19}$ Nosocomial infection was defined as infection which occurred more than 48 hours after administration or infection which occurred less than 48 hours after admission in patients who had been transferred from another hospital.

\section{Statistics}

The Mann-Whitney U-test or Student t-test were used for comparison of continuous variables. The chi-square test or Fisher exact test were used for comparison of categorical variables. p-values $<0.05$ were considered to be statistically significant. JMP version 13 (SAS Institute, Inc, Cary, NC, USA) was used for all statistical analyses.

Table 1. Patient Characteristics

\begin{tabular}{|c|c|c|c|c|c|}
\hline Characteristic & $\begin{array}{l}\text { Total episodes } \\
\qquad(\mathrm{n}=80)\end{array}$ & Group A $(n=42)^{*}$ & Group B $(n=32)^{\dagger}$ & Group $C(n=6)^{\ddagger}$ & $\mathrm{p}$-value \\
\hline Age, yr & $74.1 \pm 1.3$ & $74.3 \pm 1.8$ & $73.2 \pm 2.1$ & $78.0 \pm 4.8$ & 0.652 \\
\hline Male sex & 63 (79) & 33 (79) & $26(81)$ & $4(67)$ & 0.725 \\
\hline \multicolumn{6}{|l|}{ Comorbidity } \\
\hline Cerebrovascular disease & $19(24)$ & $9(21)$ & $9(28)$ & $1(17)$ & 0.730 \\
\hline Pulmonary disease & $5(6)$ & $3(7)$ & $2(6)$ & 0 & 0.796 \\
\hline Ischemic heart disease & $12(15)$ & $7(17)$ & $4(13)$ & $1(17)$ & 0.878 \\
\hline Liver disease & $6(8)$ & $5(12)$ & $1(3)$ & 0 & 0.280 \\
\hline Renal disease & $3(4)$ & $1(2)$ & $2(6)$ & 0 & 0.605 \\
\hline Diabetes mellitus & $20(25)$ & $15(36)$ & $4(13)$ & $1(17)$ & 0.066 \\
\hline Hypertension & $52(65)$ & $25(60)$ & $23(72)$ & $4(67)$ & 0.542 \\
\hline \multicolumn{6}{|l|}{ Profile } \\
\hline Nosocomial infection & $14(18)$ & $6(14)$ & $8(25)$ & 0 & 0.244 \\
\hline Prior hospitalization & $24(30)$ & $13(31)$ & $8(25)$ & $3(50)$ & 0.462 \\
\hline Prior use of antibiotics & $14(18)$ & $5(12)$ & $6(19)$ & $3(50)$ & 0.069 \\
\hline Prior PTBD or ERCP & $39(49)$ & $19(45)$ & $15(47)$ & $5(83)$ & 0.210 \\
\hline \multicolumn{6}{|l|}{ Laboratory data } \\
\hline Total bilirubin, mg/dL & $4.38 \pm 4.46$ & $4.26 \pm 0.68$ & $5.07 \pm 0.78$ & $1.58 \pm 1.81$ & 0.209 \\
\hline $\mathrm{CRP}, \mathrm{mg} / \mathrm{dL}$ & $6.90 \pm 6.09$ & $7.81 \pm 0.94$ & $6.22 \pm 1.07$ & $4.18 \pm 2.48$ & 0.286 \\
\hline $\mathrm{WBC}, \times 10^{3} / \mu \mathrm{L}$ & $9.55 \pm 5.43$ & $10.14 \pm 0.84$ & $8.80 \pm 0.97$ & $9.38 \pm 2.23$ & 0.579 \\
\hline Acute cholangitis severity ${ }^{\S}$ & & & & & 0.523 \\
\hline Mild & $40(50)$ & $24(57)$ & $14(44)$ & $2(33)$ & \\
\hline Moderate & $25(31)$ & $12(29)$ & $10(31)$ & $3(50)$ & \\
\hline Severe & $15(19)$ & $6(14)$ & $8(25)$ & $1(17)$ & \\
\hline Cause of biliary obstruction & & & & & 0.676 \\
\hline Stone & $40(50)$ & $24(57)$ & $14(44)$ & $2(33)$ & \\
\hline Malignancy & $38(48)$ & $17(40)$ & $17(53)$ & $4(67)$ & \\
\hline Inflammation & $2(2)$ & $1(3)$ & $1(3)$ & 0 & \\
\hline Biliary drainage method & & & & & 0.518 \\
\hline ERCP & $67(84)$ & $35(83)$ & $26(81)$ & $6(100)$ & \\
\hline PTBD & $13(16)$ & $7(17)$ & $6(19)$ & 0 & \\
\hline
\end{tabular}

Data are presented as mean \pm SD or number (\%).

PTBD, percutaneous transhepatic biliary drainage; ERCP, endoscopic retrograde cholangiopancreatography; CRP, C-reactive protein; WBC, white blood cell.

${ }^{*}$ Group A, microorganisms in both the blood and bile cultures were sensitive to the antibiotics; ${ }^{\dagger}$ Group B, only species in the blood culture were sensitive to the antibiotics; ${ }^{\ddagger}$ Group C, microorganisms in both the blood and bile cultures were insensitive to the antibiotics; ${ }^{8}$ Severity of acute cholangitis was defined according to the 2013 Tokyo Guidelines. 


\section{RESULTS}

\section{Patients}

A total of 263 episodes of acute cholangitis with either positive blood or bile culture were identified during the study period. Among those episodes, biliary drainage was successfully performed within 24 hours in 169 episodes, and either blood or bile culture was positive with sensitivity analysis available in 80 episodes (Fig. 1). Characteristics of these 80 episodes of acute cholangitis are shown in Table 1. The mean age was 74.1 years. Bile duct stones and malignant biliary obstruction were two major causes of cholangitis. Severity of cholangitis was mild in $50 \%$, moderate in 31\% and severe in 19\%. Biliary drainage was predominantly (84\%) performed by ERCP. There were three cases of inappropriate biliary drainage; two endoscopic nasobiliary drainage dislodgement and one uncontrolled cholangitis due to multiple biliary stricture. No antibiotics change was performed in any of three cases.

\section{Microbiological evaluation and antibiotics}

While the most common isolates in blood culture were $E$. coli (20\%), followed by Klebsiella (15\%), the most common isolates in bile culture were Klebsiella (50\%) and E. coli (38\%) (Table 2). All the isolates in blood culture were identified in the bile as well. Among the microorganisms detected in blood culture, Enterococcus spp. was the most common isolate resistant to the initial empiric antibiotics both in blood (5\%) and bile (24\%). The second common resistant isolate in bile was Candida spp. (14\%).

The empiric antibiotics administered are shown in Table 3. The most common empiric antimicrobial therapy was SBT/CPZ

Table 3. Initial Antibiotics Used for Acute Cholangitis

\begin{tabular}{lc}
\hline \multicolumn{1}{c}{ Antibiotics } & Episode \\
\hline $\mathrm{CMZ}$ & $2(3)$ \\
$\mathrm{SBT} / \mathrm{CPZ}$ & $37(46)$ \\
$\mathrm{CPFX}$ & $1(1)$ \\
$\mathrm{MEPM}$ & $5(6)$ \\
$\mathrm{DRPM}$ & $1(1)$ \\
$\mathrm{SBT} / \mathrm{CPZ}+\mathrm{ABPC}$ & $28(35)$ \\
SBT/CPZ+VCM & $2(3)$ \\
MEPM+ABPC & $2(3)$ \\
MEPM+LZD & $1(1)$ \\
MEPM+VCM & $1(1)$
\end{tabular}

Data are presented as number (\%).

CMZ, cefmetazole; SBT/CPZ, sulbactam/cefoperazone; CPFX, ciprofloxacin; MEPM, meropenem; DRPM, doripenem; ABPC, ampicillin; VCM, vancomycin; LZD, linezolid.

Table 2. Microorganisms Detected in Blood and Bile

\begin{tabular}{|c|c|c|c|c|}
\hline \multirow{2}{*}{ Microorganism } & \multicolumn{2}{|c|}{ Isolated microorganisms } & \multicolumn{2}{|c|}{ Resistant microorganisms } \\
\hline & Blood culture & Bile culture & Blood culture & Bile culture \\
\hline E. coli & $16(20)$ & $30(38)$ & 0 & 0 \\
\hline E. coli ESBL & 0 & 0 & 0 & 0 \\
\hline Klebsiella spp. & $12(15)$ & $40(50)$ & 0 & 0 \\
\hline Klebsiella ESBL & 0 & 0 & 0 & 0 \\
\hline Enterobacter spp. & 0 & $17(21)$ & 0 & $1(1)$ \\
\hline Pseudomonas & $4(5)$ & $8(10)$ & 0 & 0 \\
\hline Enterococcus spp. & 7 (9) & $36(45)$ & $4(5)$ & $19(24)$ \\
\hline Enterococcus faecium & $5(6)$ & $12(15)$ & $4(5)$ & 7 (9) \\
\hline Enterococcus faecalis & $1(1)$ & $18(23)$ & 0 & 7 (9) \\
\hline Other Enterococcus & $2(2)$ & $9(11)$ & 0 & $6(8)$ \\
\hline Citrobacter freundii & 0 & $6(8)$ & 0 & 0 \\
\hline Aeromonas hydrophila & $1(1)$ & $8(10)$ & $1(1)$ & $9(11)$ \\
\hline Clostridium spp. & $1(1)$ & $8(10)$ & 0 & 0 \\
\hline Bacteroides & 0 & $5(6)$ & 0 & $2(3)$ \\
\hline Staphylococcus (including MRSA) & $1(1)$ & $6(8)$ & 0 & $4(5)$ \\
\hline MRSA & $1(1)$ & $3(4)$ & 0 & $2(2)$ \\
\hline Streptococcus & 0 & $8(10)$ & 0 & 0 \\
\hline Candida spp. & 0 & $11(14)$ & 0 & $11(14)$ \\
\hline Other & $1(1)$ & $17(21)$ & $1(1)$ & $6(8)$ \\
\hline Any species & $33(41)$ & $80(100)$ & $6(8)$ & $37(46)$ \\
\hline
\end{tabular}

Data are presented as number (\%).

E. coli, Escherichia coli; ESBL, extended-spectrum $\beta$-lactamase producing species; MRSA, methicillin-resistant Staphylococcus aureus. 
(46\%), followed by SBT/CPZ and ampicillin (35\%). The empiric antibiotics were sensitive to microorganisms detected in both blood and bile culture in 42 patients (52\%, group A), only in blood culture in 32 patients (40\%, group B). In six patients, empiric antibiotics were not sensitive to microorganisms detected in blood and bile culture (group C). There were no statistical differences in baseline characteristics among three groups (Table 1). When the sensitivity of microorganisms detected in the groups drained by ERCP and percutaneous transhepatic biliary drainage was compared, there were no significant differences $(p=0.725)$ : $52 \%(35 / 67)$ and 54\% (7/13) in group A, 39\% (26/67) and 46\% $(6 / 13)$ in group $B, 9 \%(6 / 67)$ and 0 in group C.

\section{Outcomes of cholangitis according to sensitivity to em- piric antibiotics}

The overall 30-day mortality was $4 \%$ in the total cohort (Table 4), which was exclusively observed in group A, but the 30-day mortality did not differ significantly $(7 \%, 0 \%$, and $0 \%$ in groups $\mathrm{A}, \mathrm{B}$, and $\mathrm{C}, \mathrm{p}=0.244)$. There were no significant differences in other clinical outcomes, either: the mean length of hospital stay $(28.5 \pm 3.7,21.0 \pm 4.2$, and $20.5 \pm 9.8$ days in groups A, B and C, $\mathrm{p}=0.369)$, the rate of organ dysfunction $(14 \%, 25 \%$, and $17 \%$ in groups $\mathrm{A}, \mathrm{B}$ and $\mathrm{C}, \mathrm{p}=0.500)$, mean duration of fever $(4.3 \pm 0.5$, $3.2 \pm 0.6$, and $3.5 \pm 1.4$ days in groups $\mathrm{A}, \mathrm{B}$ and $\mathrm{C}, \mathrm{p}=0.921$ ) and

Table 4. Comparison of Clinical Outcomes among Three Groups According to the Pathogen Sensitivity to the Antibiotics

\begin{tabular}{|c|c|c|c|c|c|}
\hline Clinical outcome & $\begin{array}{l}\text { Total episode } \\
\quad(\mathrm{n}=80)\end{array}$ & Group A $(n=42)^{*}$ & Group B $(n=32)^{\dagger}$ & Group C $(\mathrm{n}=6)^{\ddagger}$ & p-value \\
\hline 30-day mortality & $3(4)$ & $3(7)$ & 0 & 0 & 0.244 \\
\hline Length of hospital stay, day & $24.9 \pm 24$ & $28.5 \pm 3.7$ & $21.0 \pm 4.2$ & $20.5 \pm 9.8$ & 0.369 \\
\hline Organ dysfunction & $15(19)$ & $6(14)$ & $8(25)$ & $1(17)$ & 0.500 \\
\hline Duration of fever, day & $3.8 \pm 3.5$ & $4.3 \pm 0.5$ & $3.2 \pm 0.6$ & $3.5 \pm 1.4$ & 0.921 \\
\hline Length of HCU stay, day & $1.3 \pm 2.9$ & $1.4 \pm 0.4$ & $1.2 \pm 0.5$ & $1.7 \pm 1.2$ & 0.070 \\
\hline
\end{tabular}

Data are presented as number $(\%)$ or mean \pm SD.

HCU, high care unit.

${ }^{*}$ Group A, microorganisms in both the blood and bile cultures were sensitive to the antibiotics; ${ }^{\dagger}$ Group B, only species in the blood culture were sensitive to the antibiotics; ${ }^{\ddagger}$ Group C, microorganisms in both the blood and bile cultures were insensitive to the antibiotics.

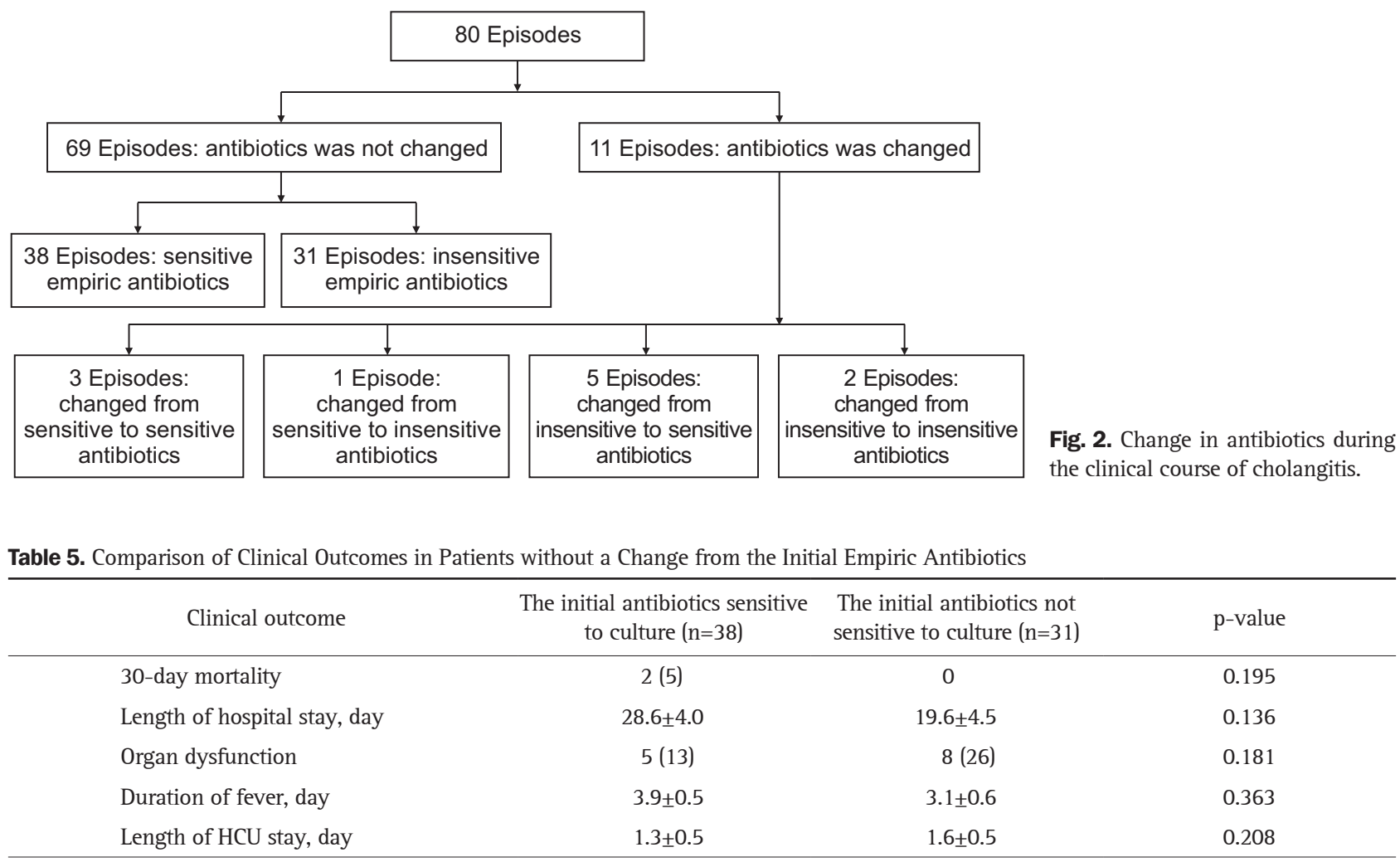

Data are presented as number (\%) or mean \pm SD.

HCU, high care unit. 
mean duration of high care unit stay $(1.4 \pm 0.4,1.2 \pm 0.5$, and $1.7 \pm 1.2$ days in groups $\mathrm{A}, \mathrm{B}$ and $\mathrm{C}, \mathrm{p}=0.070$ ).

\section{Clinical outcomes by the antibiotics change}

During the clinical course of 80 episodes of acute cholangitis, the initial empiric antibiotics were changed in 11 episodes (Fig. 2). Among the remaining 69 episodes of cholangitis without antibiotics change, the initial empiric antibiotics were sensitive in 38 episodes and insensitive in 31 episodes. As shown in Table 5 , sensitivity of antibiotics to microorganisms detected in blood or bile culture did not appear to be associated with clinical outcomes, which suggested that sensitivity of empiric antibiotics is not clinically relevant once adequate biliary drainage is achieved for acute cholangitis. Antibiotics given in two mortal cases were sensitive to microorganisms detected in blood and bile culture.

The initial empiric antibiotics were changed in 11 episodes because of the lack of immediate clinical response in seven and despite clinical response in four. Among seven cases without immediate clinical response, antibiotics were changed according to the microorganisms detected in culture only in three cases. In the remaining four cases without clinical response, antibiotics were changed empirically but antibiotics change was eventually unnecessary or inappropriate in terms of sensitivity: Sensitive to sensitive ones in two, sensitive to insensitive ones in one and insensitive to insensitive ones in one. Meanwhile, among four cases with antibiotics change despite clinical response, three cases underwent antibiotics change according to the microbiological analysis, which turned out to be sensitive to sensitive ones. In the remaining one case undergoing antibiotics change for an unknown reason despite clinical response, both the initial and second antibiotics were insensitive to Bacillus and Rhizobium detected in the bile. In summary, antibiotics were changed from sensitive to sensitive ones in three, sensitive to insensitive ones in one and insensitive to sensitive ones in five and insensitive to insensitive ones in two (Fig. 2). In three cases who received insensitive antibiotics even after antibiotics change, the clinical courses of cholangitis were not complicated by the organ function or mortality.

\section{DISCUSSION}

While acute cholangitis is managed by a combination of biliary drainage and antibiotics, our retrospective study results suggested that sensitivity of empiric antibiotics to microorganisms detected in blood or bile was not associated with clinical outcomes of acute cholangitis after successful biliary drainage. Empiric antibiotics were only sensitive to blood culture in $40 \%$ and not sensitive to bile and blood culture in $8 \%$ but there was no significant difference in 30-day mortality in those cases. There were no significant differences in other clinical outcomes such as organ dysfunction and length of hospital stay, either.
Although severe acute cholangitis accounted for 19\% of our study cohort, the 30-day mortality rate was only 4\%, which was exclusively observed in patients who received antibiotics sensitive to microorganisms detected in blood and bile culture.

Among 11 episodes of antibiotics change, antibiotics regimens were changed appropriately (from insensitive to sensitive ones) only in five episodes according to the sensitivity analyses. In the remaining six episodes when antibiotics were not changed based on the sensitivity analyses, antibiotics change was unnecessary or inappropriate (Fig. 2). Therefore, empiric antibiotics should be changed only after antibiotics sensitivity is available. Meanwhile, since sensitivity of empiric antibiotics did not affect clinical outcomes significantly in cases without antibiotics change (Table 5), in cases with clinical response antibiotics change might not be necessary irrespective of sensitivity of the initial antibiotics.

Some studies recommended bile culture should be performed at the time of biliary drainage because bile culture is more polymicrobial than blood culture, based on the assumption that all microorganisms detected in the bile should be covered by antibiotics. ${ }^{15-17}$ However, clinical outcomes were not discussed in those studies. To the best of our knowledge, this is the first study to compare clinical outcomes according to empiric antibiotics sensitivity to blood and bile culture. Our study suggested that covering all the microorganisms in bile or blood might not be necessarily mandatory in cases with acute cholangitis after successful biliary drainage. However, our study results did not indicate that bile or blood culture is unnecessary in the management of cholangitis. We recommend selecting appropriate antibiotics once susceptibility is available to better manage cholangitis and to avoid further pandemic of resistant bacteria.

In general, insensitive antibiotics for infectious diseases are related to poor clinical outcomes but it depends on primary infection sites. ${ }^{20,21}$ The mortality rate of biliary tract infection is relatively low probably because of biliary decompression. ${ }^{15}$ In our study, among 11 cases with antibiotics switch, empiric antibiotics were changed to insensitive ones in three cases but it did not adversely affect clinical outcomes in cholangitis. In line with previous reports, our findings imply that the role of biliary drainage is indispensable in the management of cholangitis.

Several limitations of this study should be acknowledged. First, this is a single center, retrospective study and a largescale, prospective study is warranted. It should be confirmed whether the use of insensitive antibiotics does not increase rare systemic complications such as infective endocarditis and liver abscess. Of note, in our study cohort, Klebsiella and E. coli are the two major microorganisms and Enterococcus was detected only in 9\% of blood culture. In cases with Enterococcal bacteremia, antibiotics should be changed to prevent Enterococcal infective endocarditis according to the recommendations in TG13. ${ }^{22}$ Although there were no extended-spectrum $\beta$-lactamase-producing $E$. coli and Klebsiella detected in our 
study cohort, they are increasingly detected in bile culture nowadays. Therefore, large-scale data should be further collected in a multicenter study to evaluate generalizability of our study results. In addition, in some cases with hilar biliary stricture, biliary drainage can be incomplete and antibiotics sensitivity can affect clinical outcomes in those cases. Furthermore, we evaluated the length of hospital stay as one of study endpoints, but some patients had second ERCP for stone extraction in cases with bile duct stones or exchange from endoscopic nasobiliary drainage to stents in case with malignant biliary obstruction. Therefore, the length of hospital stay can be affected not only by clinical response of cholangitis but by the primary causes of cholangitis. Lastly, our study cohort included only those cases who underwent biliary drainage within 24 hours of the onset of cholangitis. However, early biliary drainage is not always available in some centers and elective biliary drainage is performed after the initial empiric antibiotics use. The results of bile culture might be different in those cases undergoing elective biliary drainage compared to our study results.

In conclusion, the sensitivity of empiric antibiotics was not associated with clinical outcomes in patients with acute cholangitis. Our study results suggest that covering all microorganisms detected in blood and bile culture is not always necessary to resolve acute cholangitis once adequate biliary drainage is achieved. However, in cases without clinical response even after biliary drainage, sensitivity of microorganisms detected in bile and blood culture should be evaluated and antibiotics should be changed based on the sensitivity analysis if necessary. Finally, since this is a single center, retrospective study, our study results should be further validated in a large-scale multicenter study.

\section{CONFLICTS OF INTEREST}

No potential conflict of interest relevant to this article was reported.

\section{AUTHOR CONTRIBUTIONS}

Study concept and design: S.K., N.T. Data acquisition: S.K., Y.K. Data analysis and interpretation: S.K., N.T., Y.N. Drafting of the manuscript: N.T., Y.N. Critical revision of the manuscript for important intellectual content: K. Koike, K.T. Technical and material support: C.S., K. Kurokawa, J.A., K.F., S.K., Y.K., S.M., M.K., K. Kojima, T.O., M.S. All authors contributed to critical revision and approved of the final version.

\section{ORCID}

Satoshi Kawamura

Yuki Karasawa

Nobuo Toda

Yousuke Nakai https://orcid.org/0000-0002-7031-5388

https://orcid.org/0000-0001-8278-7641

https://orcid.org/0000-0001-5426-1896

https://orcid.org/0000-0001-7411-1385
Chikako Shibata

https://orcid.org/0000-0001-9492-3794

Ken Kurokawa

Junya Arai

Kazuyoshi Funato

Shigeyuki Kurosaki

Shuya Maeshima

Mayuko Kondo

Kentaro Kojima

Takamasa Ohki

Michiharu Seki

Kazuhiko Koike

Kazumi Tagawa

\section{REFERENCES}

1. Lai EC, Mok FP, Tan ES, et al. Endoscopic biliary drainage for severe acute cholangitis. N Engl J Med 1992;326:1582-1586.

2. Leung JW, Chung SC, Sung JJ, Banez VP, Li AK. Urgent endoscopic drainage for acute suppurative cholangitis. Lancet 1989;1:1307-1309.

3. Dooley JS, Hamilton-Miller JM, Brumfitt W, Sherlock S. Antibiotics in the treatment of biliary infection. Gut 1984;25:988-998.

4. Isayama H, Yasuda I, Tan D. Current strategies for endoscopic management of acute cholangitis. Dig Endosc 2017;29 Suppl 2:70-77.

5. Umefune G, Kogure H, Hamada T, et al. Procalcitonin is a useful biomarker to predict severe acute cholangitis: a single-center prospective study. J Gastroenterol 2017;52:734-745.

6. Sung YK, Lee JK, Lee KH, Lee KT, Kang CI. The clinical epidemiology and outcomes of bacteremic biliary tract infections caused by antimicrobial-resistant pathogens. Am J Gastroenterol 2012;107:473-483.

7. Ibrahim EH, Sherman G, Ward S, Fraser VJ, Kollef MH. The influence of inadequate antimicrobial treatment of bloodstream infections on patient outcomes in the ICU setting. Chest 2000;118:146155

8. Harbarth S, Garbino J, Pugin J, Romand JA, Lew D, Pittet D. Inappropriate initial antimicrobial therapy and its effect on survival in a clinical trial of immunomodulating therapy for severe sepsis. Am J Med 2003;115:529-535.

9. Kang CI, Kim SH, Park WB, et al. Bloodstream infections caused by antibiotic-resistant gram-negative bacilli: risk factors for mortality and impact of inappropriate initial antimicrobial therapy on outcome. Antimicrob Agents Chemother 2005;49:760-766.

10. Paul M, Kariv G, Goldberg E, et al. Importance of appropriate empirical antibiotic therapy for methicillin-resistant Staphylococcus aureus bacteraemia. J Antimicrob Chemother 2010;65:2658-2665.

11. Suppli M, Aabenhus R, Harboe ZB, Andersen LP, Tvede M, Jensen JU. Mortality in enterococcal bloodstream infections increases with inappropriate antimicrobial therapy. Clin Microbiol Infect 2011;17:1078-1083.

12. Tagashira $\mathrm{Y}$, Sakamoto $\mathrm{N}$, Isogai $\mathrm{T}$, et al. Impact of inadequate ini- 
tial antimicrobial therapy on mortality in patients with bacteraemic cholangitis: a retrospective cohort study. Clin Microbiol Infect 2017;23:740-747.

13. Royo-Cebrecos C, Gudiol C, García J, et al. Characteristics, aetiology, antimicrobial resistance and outcomes of bacteraemic cholangitis in patients with solid tumours: a prospective cohort study. J Infect 2017;74:172-178.

14. Kang CI, Sung YK, Lee KH, Lee KT, Lee JK. Clinical impact of inappropriate initial antimicrobial therapy on outcome in bacteremic biliary tract infections. Scand J Infect Dis 2013;45:227-234.

15. Lee CC, Chang IJ, Lai YC, Chen SY, Chen SC. Epidemiology and prognostic determinants of patients with bacteremic cholecystitis or cholangitis. Am J Gastroenterol 2007;102:563-569.

16. Negm AA, Schott A, Vonberg RP, et al. Routine bile collection for microbiological analysis during cholangiography and its impact on the management of cholangitis. Gastrointest Endosc 2010;72:284-291.

17. Rupp C, Bode K, Weiss KH, et al. Microbiological assessment of bile and corresponding antibiotic treatment: a strobe-compliant observational study of 1401 endoscopic retrograde cholangiogra- phies. Medicine (Baltimore) 2016;95:e2390.

18. Kiriyama S, Takada T, Strasberg SM, et al. TG13 guidelines for diagnosis and severity grading of acute cholangitis (with videos). $\mathrm{J}$ Hepatobiliary Pancreat Sci 2013;20:24-34.

19. Kogure H, Tsujino T, Yamamoto K, et al. Fever-based antibiotic therapy for acute cholangitis following successful endoscopic biliary drainage. J Gastroenterol 2011;46:1411-1417.

20. Joo EJ, Kang CI, Ha YE, et al. Impact of inappropriate empiric antimicrobial therapy on outcome in Pseudomonas aeruginosa bacteraemia: a stratified analysis according to sites of infection. Infection 2011;39:309-318.

21. Kang CI, Song JH, Chung DR, et al. Clinical impact of methicillin resistance on outcome of patients with Staphylococcus aureus infection: a stratified analysis according to underlying diseases and sites of infection in a large prospective cohort. J Infect 2010;61:299-306.

22. Gomi H, Solomkin JS, Takada T, et al. TG13 antimicrobial therapy for acute cholangitis and cholecystitis. J Hepatobiliary Pancreat Sci 2013;20:60-70. 